\title{
Fragmentum Cracoviense Officii Sancti Demetrii Thessalonicensis ${ }^{1}$
}

\author{
Zsuzsa CZAGÁnY \\ Institut für Musikwissenschaft \\ Forschungszentrum für Humanwissenschaften \\ Ungarische Akademie der Wissenschaften \\ Táncsics Mihály u. 7, H-1014 Budapest, Ungarn \\ E-Mail: czagany.zsuzsa@btk.mta.hu
}

(Angekommen: Februar 2015; angenommen: April 2015)

\begin{abstract}
The manuscript Ms. 2372 of the Jagiellonian Library in Kraków contains a notated fragment of an early $12^{\text {th }}$-century Hungarian antiphoner as a flyleaf. The text written with late Caroline Minuscule is accompanied by two types of music notation. The recitative and formulaic chants appearing in the rubrics (responsoria brevia and versicles of the horae minores) are notated with diastematic neumes. The main text containing the antiphons and responsoria prolixa is written with what is probably the earliest example of the medieval Hungarian (Strigonian) chant notation. The fragment is also remarkable because of its unique content. While the recto contains the standard repertory of the office for St Michael, the verso includes chants from the historia Perennis patrie regis of St Demetrius of Thessaloniki venerated in medieval Hungary as one of its patron-saints. The Cracovian fragment represents the oldest testimonial of the historia discovered so far.
\end{abstract}

Keywords: antiphoner, historia, St Demetrius of Thessaloniki, Hungarian Strigonian Chant Notation

\section{Beschreibung und Inhalt des Fragments}

Im folgenden Beitrag wird das im Einband der Handschrift 2372 der Biblioteka Jagiellońska in Krakau enthaltene notierte Fragment eines vermutlich in Ungarn entstandenen Antiphonars behandelt. Für den Hinweis auf das Fragment sowie die Zusendung seiner digitalen Kopien bin ich Herrn Dr. Jakub Kubieniec vom

1. Folgender Beitrag wurde im Rahmen des Projektes NK 104426 des National Research, Development and Innovation Fund (früher: Ungarischer Förderungsfonds der Wissenschaftlichen Forschung - OTKA) vorbereitet. 
Institut für Musikwissenschaft der Jagiello Universität in Krakau zum Dank verpflichtet.

Die Trägerhandschrift wird im klassischen Katalog von Władysław Wisłocki beschrieben, der den Bestand der mittelalterlichen Handschriften der Jagiellonischen Bibliothek zwischen 1877 und 1881 inventarisierte. ${ }^{2}$ Im Gegensatz zu den eher spärlichen kodikologischen und buchgeschichtlichen Informationen sind die inhaltlichen Beschreibungen ausführlicher. Demgemäß handelt es sich bei Ms. 2372 um eine theologische Sammelhandschrift des 15. Jahrhunderts ${ }^{3}$ hauptsächlich mit Werken zeitgenössischer Autoren - überwiegend Professoren der theologischen Fakultät der Krakauer Universität. ${ }^{4}$ Sie wurde offensichtlich im Umkreis der Universität zusammengestellt und als Lehrtext im Unterrichtsprozess benutzt. Abschrift und Zusammenstellung des Kompendiums stammen von Johannes Cantius (1390-1473), dem hochgeschätzten Professor der Theologie und Dekan der Artistenfakultät in Krakau, der 1676 seliggesprochen und 1767 kanonisiert wurde. ${ }^{5}$

Das nun zu besprechende Antiphonarfragment ist als Vorsatzblatt der Sammelhandschrift überliefert. Da es nicht auf den Einbanddeckel aufgeklebt wurde, sind beide Seiten sichtbar und erforschbar.

Das beidseitig beschriebene Pergamentblatt enthält 13 Textzeilen mit Choralnotation in einem Vierliniensystem (verso) bzw. 10 notierte Textzeilen und 6 Textzeilen mit linienloser Neumenschrift (recto) in einspaltiger Anlage (Abbildung 1-2). Es ist oben und unten abgeschnitten. Das ursprüngliche Folio dürfte auf beiden Enden mindestens je eine Zeile mehr enthalten haben. Wegen des Beschneidens bei der Einbindung sind die Zeilen ebenfalls unvollständig. Die synoptische Übersicht des Inhalts von Recto und Verso zeigen die Tabellen 1-2. ${ }^{6}$

Auf der Recto-Seite sind Teile des Michaelsoffiziums überliefert: Die Gesänge der laudes, der horae minores sowie der 2. Vesper sind zwar wegen des abgeschnittenen inneren Blattrandes unvollständig, ihre Zusammenstellung und Reihenfolge jedoch rekonstruierbar. Die detailliert ausgeführte Anlage des Offiziums mit Angabe sämtlicher responsoria brevia und Versikel der kleinen Horen sowie mit mehreren, der Magnificat-Antiphon der Vesper beigefügten überzähligen Antiphonen findet sich in zahlreichen ungarischen Offiziumshandschriften. Obwohl das

2. Władysław Wisłocki, Catalogus Codicum Manuscriptorum Bibliothecae Universitatis Jagellonicae Cracoviensis (Cracoviae: Typis Universitatis Jagellonicae, 1877-1881), Bd. 2, 565.

3. Varii Tractatus theologici, Quaestiones et Sermones, scripti manu b. Joannis Cantii [...], in Wislocki, Catalogus, 565.

4. Paulus de Zathor, Nicolaus Koslowsky, Matheus de Cracovia.

5. Zu Person und Schrifttum des Johannes Cantius siehe Ryszard Jan Zawadzki, „Studia teologiczne sw. Jana Kantego“, Analecta Cracoviensia 25 (1993), 551-568; ders., „Der handschriftliche Nachlass des hl. Johannes Cantius“, in Die Anfänge des Schrifttums in Oberschlesien bis zum Frühhumanismus, hrsg. von Gerhard Kosellek (Frankfurt am Main: Lang, 1997), 125-137.

6. Die rekonstruierten Textteile erscheinen in eckigen Klammern. Bei der Bestimmung der liturgischen Gattungen wurden folgende Abkürzungen verwendet: $\mathrm{a} / \mathrm{A}=\mathrm{Antiphon} ; \mathrm{Ab}=$ antiphona ad Benedictus; $\mathrm{Am}=\mathrm{an}-$ tiphona ad Magnificat; $\mathrm{r}=$ responsorium breve; $\mathrm{R}=$ responsorium prolixum; $\mathrm{V}=$ versus responsorii; $\mathrm{W}=\mathrm{versicu}-$ lus; $\mathrm{H}=$ Hymnus. 
AbBILDUng 1 Ms. 2372 / recto

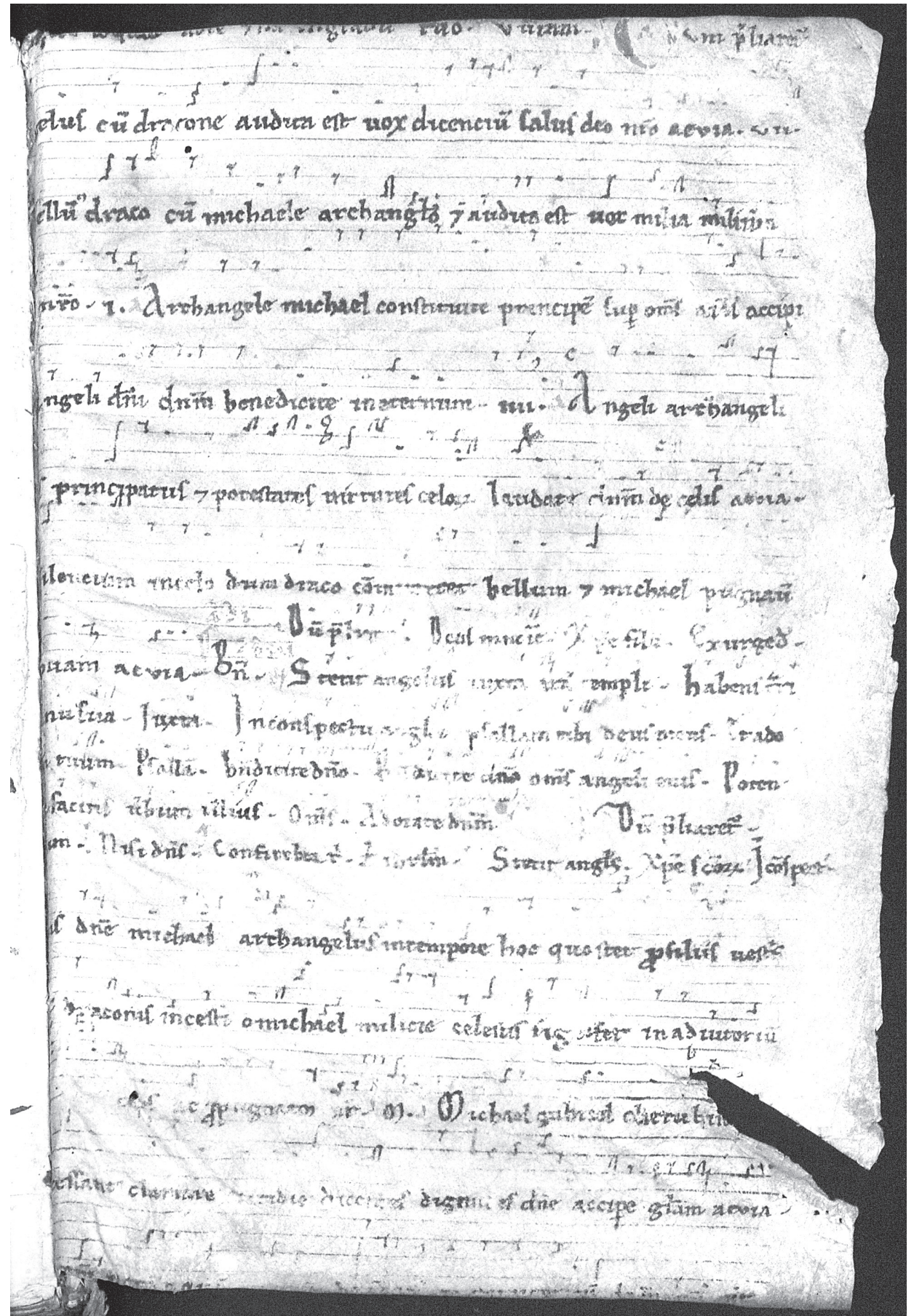


ABBiLdung 2 Ms. 2372 / verso

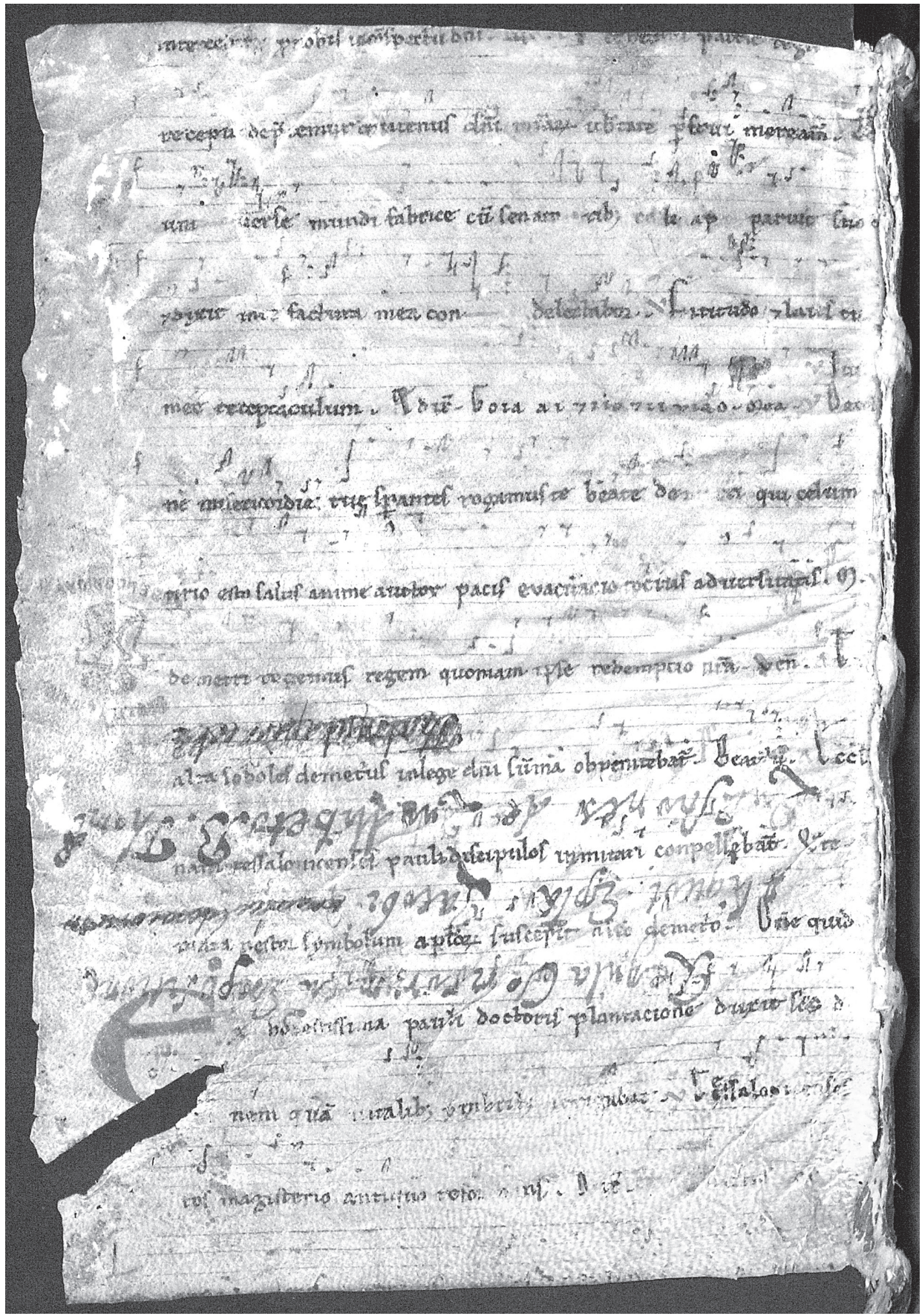


TABELLE 1 Ms. 2372 / recto: [Michaelis]

\begin{tabular}{|c|c|}
\hline \multirow[t]{7}{*}{\begin{tabular}{|l} 
[n laudibus] \\
\end{tabular}} & \\
\hline & a1 Dum preliaretur \\
\hline & a2 [Dum committeret] bellum \\
\hline & a3 Archangele Michael \\
\hline & a4 Angeli Domini \\
\hline & a5 Angeli Archangeli \\
\hline & $\mathrm{Ab}$ [Factum est] silencium \\
\hline \multirow[t]{4}{*}[\mathrm{Ad}\mathrm{I}]{} & \\
\hline & a Dum preliaretur \\
\hline & r Christe fili \\
\hline & W Exurge Domine \\
\hline \multirow[t]{2}{*}{ [Ad III] } & \\
\hline & r Stetit angelus \\
\hline \multirow[t]{3}{*}[\mathrm{Ad}\mathrm{VI}]{} & \\
\hline & r In conspectu angelorum \\
\hline & W Benedicite Domino \\
\hline \multirow[t]{3}{*}{ [Ad IX] } & \\
\hline & r Benedicite Domino \\
\hline & W Adorate Dominum \\
\hline \multicolumn{2}{|c|}{ [In secundis vesperis] } \\
\hline & aa Dum preliaretur [...] \\
\hline & R Stetit angelus \\
\hline & H Christe sanctorum \\
\hline & W In conspectu \\
\hline & Am [Consurgat quesumu]s Domine \\
\hline \multicolumn{2}{|c|}{ A+ Michael Gabriel cherubim } \\
\hline \multicolumn{2}{|c|}{ 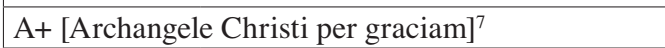 } \\
\hline
\end{tabular}

Michaelsoffizium zum klassischen Bestand des europäischen Choralrepertoires gehört, zeigt sich in Auswahl und Zusammenstellung von Kurzresponsorien und Versikeln der kleinen Horen eine recht große Variabilität in den einzelnen Traditionen, ja sogar unter Handschriften, die zum selben Überlieferungskreis gehören. ${ }^{8}$

7. Der Text der Antiphon Archangele Christi per graciam am unteren Blattrand des Recto ist nicht mehr lesbar, die Antiphon kann aber aufgrund der erhaltenen Melodiezeile mit Sicherheit identifiziert werden.

8. Die unterschiedlichen Redaktionen des Michaelsoffiziums in den mittelalterlichen ungarischen Überlieferungskreisen siehe in Corpus Antiphonalium Officii Ecclesiarum Centralis Europae (im Weiteren: $C A O-E C E)$ V/B Esztergom (Sanctorale), hrsg. von Andrea Kovács (Budapest: Institute for Musicology of the Hungarian Academy of Sciences, 2006), 154-155, 174-175; CAO-ECE VI/B Kalocsa-Zagreb (Sanctorale), hrsg. von Andrea Kovács (Budapest: Institute for Musicology of the Hungarian Academy of Sciences, 2008), 124-126, 143-144; CAO-ECE VII/B Transylvania-Várad (Sanctorale), hrsg. von Andrea Kovács (Budapest: Institute for Musicology of the Hungarian Academy of Sciences and Research Group for Church Music at the Liszt Ferenc Academy of Music, 2010), 116-118. 
TABelLe 2 Ms. 2372 / verso: [Michaelis; Demetrii]

\begin{tabular}{|c|c|}
\hline $\mathrm{A}+[$ Michael Ga & briel Raphael summi nuncii...] intercedite \\
\hline [Demetrii / in p & rimis vesperis] \\
\hline & a Perennis patrie regis \\
\hline & R C [onditor] universe mundi \\
\hline & V Fortitudo et laus tua \\
\hline & H Deus tuorum militum \\
\hline & W Iustus ut palma (?) \\
\hline & Am [In multitudi]ne misericordie \\
\hline [Invitatorium] & \\
\hline & a [Preciosi martyris] Demetrii rogemus \\
\hline [In primo noctu & rno] \\
\hline & a1 T[eneris sub annis] \\
\hline & a2 Eccle[siarum discipli]nam \\
\hline & a3 [Intestina fidei dog]mata \\
\hline & R1 Ex honestissima Pauli \\
\hline & V1 Thesalonicenses [ex electionis] \\
\hline & R2 Dilectus [Dei Demetrius] \\
\hline
\end{tabular}

Im Gegensatz zu dieser Unbeständigkeit erscheint die Redaktion des Michaelsoffiziums, die in den späteren Handschriften von Esztergom/Gran vor uns tritt, als überaus einheitlich. Das Fragment weicht von dieser ausgeprägten Gestalt ab, stimmt aber mit der Redaktion einiger um 1100 verfassten süddeutschen Antiphonare überein. ${ }^{9}$ Die Frage, ob es sich bei dieser auf der Mikroebene greifbaren Übereinstimmung um einen Zufall, eine individuelle Auswahl oder eine direkte, bewusste Übernahme aus dem süddeutschen Gebiet handelt, muss angesichts des schmalen Quellenbestandes vorerst offenbleiben.

Obwohl die zweite Vesper anscheinend vollständig ist, geht das Michaelsoffizium mit der letzten Zeile des Recto noch nicht zu Ende. Nach der Magnificat-Antiphon Consurgat quesumus Domine folgen weitere überzählige Antiphonen (Michael Gabriel cherubim, Archangele Christi per graciam), die offensichtlich für die Oktavtage bestimmt waren. Die schwer lesbare oberste Zeile auf der VersoSeite verrät sogar die Anwesenheit einer dritten, ebenfalls zum Michaelsoffizium gehörenden Antiphon (Michael Gabriel Raphael summi nuncii), unmittelbar gefolgt von der Eröffnungsantiphon der ersten Vesper des Demetriusoffiziums. ${ }^{10}$ Das Nacheinanderfolgen der beiden Offizien - Michael und Demetrius - hat

9. Vgl. Antiphonar aus Klosterneuburg, Bibliothek des Augustiner-Chorherrenstiftes 1012, f. 81v-82r. Zur Überlieferung in den salzburgischen Quellen vgl. CAO-ECE I/B Salzburg (Sanctorale), hrsg. von Judit Fehér und László Dobszay (Budapest: Institute for Musicology of the Hungarian Academy of Sciences, 2009), 112.

10. [...] intercedite pro nobis in conspectu Domini. 
schwerwiegende Konsequenzen, die zum Schluss des Beitrags ausführlich besprochen werden sollen.

Außer der letzten, noch zum Michaelsoffizium gehörenden Antiphon in der obersten erhaltenen Zeile wird das vollständige Verso von Gesängen des Demetriusoffiziums gefüllt. Abgesehen von der Beschädigung der oberen und unteren Blattränder sowie des rechten Seitenrandes, die bei der Neuverwendung des Pergaments abgeschnitten wurden, ist die erste Vesper vollständig mit antiphona sola, Responsorium mit voll ausgeschriebenem Versus und Doxologie, rubriziertem Hymnus und Versikel sowie Magnificat-Antiphon. Das Invitatorium mit den drei Antiphonen der ersten Nokturn der Matutin kann ebenfalls vollständig rekonstruiert werden. Von den drei darauffolgenden Responsorien ist dagegen nur das Erste mit Sicherheit zu erschließen. Das Zweite ist lediglich durch das rubrikale R und das stark verblasste Anfangswort Dilectus bestimmbar.

Wie es in der historischen, hagiographischen und neulich auch musikwissenschaftlichen Forschung bekannt ist, hatte der in der Ostkirche besonders verehrte heilige Demetrius im Mittelalter einen festen Platz in der Reihe der ungarischen Landesheiligen eingenommen. ${ }^{11}$ Laut der allgemein akzeptierten Meinung liegen die Gründe dafür in der pannonischen (sirmianischen) Herkunft des Demetriuskultes. Die im beginnenden 5. Jahrhundert bereits in einem weiten Umkreis bekannte Verehrung wurde erst nach der Eroberung von Sirmium im Jahre 442 durch die Hunnen zum zweiten, östlichen Zentrum Thessaloniki übertragen. In den Passionen, die als Ergebnis des neu aufsteigenden Demetriuskultes entstanden, wurden Herkunft und Wurzel des Heiligen verschleiert, um ihn nunmehr als einen thessalonischen Stadtheiligen betrachten zu können.

Die Anwesenheit von Demetrius ist zwar in den liturgischen Büchern des mittelalterlichen Ungarn nicht derart konsequent wie die der dynastischen Heiligen der Arpaden, zeigt aber in der ,zweiten Linie“ des proprium hungaricum ab dem frühen 12. Jahrhundert bis zum Spätmittelalter eine grundsätzliche Kontinuität. Die Untersuchung von Genese und Quellen der ungarischen Demetriuslegende führte Péter Tóth zur Ansicht, in der Geschichte der ungarischen Demetriustradition seien drei Schichten bzw. voneinander auch zeitlich abgrenzbare Perioden zu bestimmen. ${ }^{12}$ Die Legende selbst wurde nach der Meinung von Tóth in der letzten Periode dieses vom ausgehenden 11. bis zur Wende des 12.-13. Jahrhunderts dauernden Zeitraums erschaffen. Das Demetriusoffizium konnte dagegen anhand von philologischen und musikgeschichtlichen Beobachtungen etwas früher, in die erste Hälfte (um die Mitte) des 12. Jahrhunderts, datiert werden. Philologische

11. Zur Geschichte der Demertriusverehrung in Ungarn mit Zusammenfassung der bisherigen Forschungsergebnisse und Literaturübersicht vgl. Zsuzsa Czagány - Péter Tóth, Historia sancti Demetrii Thessalonicensis (Lions Bay: Institute of Mediæval Music, 2013) (=Wissenschaftliche Abhandlungen / Musicological Studies, series HISTORIAE 65/20), ix-xxii.

12. Ebd., xviii. 
Argumente dafür liegen in der Tatsache, dass die Texte der Antiphonen und Responsorien weder sprachliche noch inhaltliche Beziehungen zum Legendentext aufweisen. Unter anderem lassen sie die in der Legende stark betonte pannonische Herkunft des Heiligen außer Acht. ${ }^{13}$ Von Seiten der musikalischen Untersuchung sprechen wiederum die Variabilität der Anlage und die Zusammenstellung des Repertoires sowie die melodischen Eigenschaften der Gesänge für einen archaischen Zustand des Demetriusoffiziums. ${ }^{14}$

Das Offizium oder die Historia Perennis patriae regis gilt demnach als das früheste greifbare Denkmal der mittelalterlichen ungarischen Demetriusverehrung. Ähnlich wie bei zahlreichen mittelalterlichen Heiligenoffizien setzt aber seine handschriftliche - sowohl textliche als auch musikalische - Überlieferung viel später ein als seine vermutete Entstehungszeit (Tabelle 3).

Die früheste handschriftliche Eintragung der Historia findet sich in dem heute in Zagreb aufbewahrten Breviarium Strigoniense des späten 13. Jahrhunderts, ${ }^{15}$

TABelle 3 Verzeichnis der Quellen des Demetriusoffiziums

\begin{tabular}{|l|l|l|}
\hline Breviarium Strigoniense & s.13/2 & Zagreb, Metropolitanska knjižnica MR 67 \\
\hline Breviarium Strigoniense & 1484 & (impr.) Nürnberg (G. Stuchs) \\
\hline Antiphonale Strigoniense & $\mathrm{c} 1360$ & Istanbul, Topkap Saray, Deissmann 42 \\
\hline Antiphonale Scepusiense & $\mathrm{s} .15$ & Knižnica Spišskej kapituly Ms. Mus. No. 2. \\
\hline $\begin{array}{l}\text { Breviarium Strigoniense aus } \\
\text { Bártfa (Bardejov) }\end{array}$ & $\mathrm{s} .15$ & $\begin{array}{l}\text { Budapest, Magyar Nemzeti Múzeum } \\
\text { (Ungarisches Nationalmuseum) 63.74.1.C. }\end{array}$ \\
\hline $\begin{array}{l}\text { Breviarium Strigoniense aus } \\
\text { Bártfa (Bardejov) }\end{array}$ & $\mathrm{s} .15$ & $\begin{array}{l}\text { Budapest, Magyar Nemzeti Múzeum } \\
\text { (Ungarisches Nationalmuseum) 63.84.C. }\end{array}$ \\
\hline $\begin{array}{l}\text { Breviarium Strigoniense (aus dem } \\
\text { Gebiet der heutigen Slowakei) }\end{array}$ & $\mathrm{s.15}$ & $\begin{array}{l}\text { Alba Iulia, Biblioteca Naţională a României-filiala } \\
\text { Batthyaneum R III 40 }\end{array}$ \\
\hline Breviarium Waradiense & 1460 & Roma, Biblioteca Apostolica Vaticana 8247 \\
\hline Breviarium aus Siebenbürgen & s.15 & Güssing, Klosterbibliothek der Franziskaner 1/34 \\
\hline Antiphonale Waradiense (fragm.) & s.15/ex & $\begin{array}{l}\text { Budapest, Országos Széchényi Könyvtár } \\
\text { (Széchényi Nationalbibliothek) A 58 }\end{array}$ \\
\hline Breviarium Strigoniense & s.15 & $\begin{array}{l}\text { Alba Iulia, Biblioteca Naţională a României-filiala } \\
\text { Batthyaneum R I 110 }\end{array}$ \\
\hline Breviarium Zagrebiense & 1484 & (impr.) Venedig (E. Ratdolt) \\
\hline
\end{tabular}

13. „Dieser relativ häufig vorkommende Text [des Offiziums] scheint über die Einzelheiten der Demetriuspassion, wie Nestor, Lyaeus, Saloniki und die dortigen Wundererscheinungen (mit Ausnahme des Myrons), informiert zu sein, gibt aber keinerlei Hinweise für die heimische, pannonische Herkunft des Heiligen. Noch mehr verwundert, dass an jenen Parallelstellen, die sowohl in der Legende als auch im Offizium erscheinen, keinerlei Überlappungen zu finden sind: Textverlauf, Wortschatz und Stil scheinen völlig unabhängig von der Legende konzipiert worden zu sein." Ebd., xviii.

14. Hingewiesen sei weiter auf das schwankende Verhältnis der Commune- und Propriumsstücke, die Miteinbeziehung und „Aktualisierung“ von Commune-artigen Antiphonen und Responsorien, die an einer Vielzahl von Festen ähnlichen Charakters gesungen werden konnten, kurz: auf den Mangel einer greifbaren proprialen Grundgestalt. Mehr dazu ebd., xxxii-xxxiv.

15. Zagreb, Metropolitanska knjižnica MR 67, f. 303v-305r. 
das jedoch nur die Texte der Gesänge überliefert. Die notierte Fassung ist in drei Handschriften belegt, von denen allerdings einzig das auf dem Gebiet der heutigen Slowakei entstandene Antiphonale Scepusiense des 15. Jahrhunderts den vollständigen Zyklus enthält. ${ }^{16}$ Die zwei anderen Quellen überliefern lediglich Teile des Offiziums. Das um 1360 geschriebene, heute in Istanbul aufbewahrte Antiphonale Strigoniense enthält im Nachtrag Bruchstücke der beiden Vespern, ${ }^{17}$ das Fragment des dem südöstlichen Ritusgebiet gehörenden Antiphonale Waradiense wiederum Ausschnitte zweier Responsorien der Matutin. ${ }^{18}$ Der früheste vollwertige musikalische Beleg für die Historia ist demnach etwa 250 Jahre später entstanden als das Offizium selbst. Durch das neu aufgefundene und hier erstmals vorgelegte Fragment der Krakauer Biblioteka Jagiellońska kommt es zur entscheidenden Änderung dieser spärlichen Quellenlage. Es geht nicht einfach um Quellenzuwachs: Das polnische Fragment ist die älteste bisher bekannte Quelle der ungarischen Demetrius-Historia. Ihre Entstehung liegt in unmittelbarer zeitlicher Nähe zur vermuteten, durch Primärquellen bisher aber nicht belegten Entstehungszeit des Zyklus.

\section{Schrift und Notation}

Die Schrift des Fragments repräsentiert eine Spätform der karolingischen Minuskel im Frühstadium der Gotisierung. Das Schriftbild scheint dem der frühesten ungarischen Offiziumshandschrift, dem im 12. Jahrhundert geschriebenen Codex Albensis, nahe zu stehen. ${ }^{19}$ Laut László Mezey gehört die Skriptur dieses Antiphonars noch in die letzte, dem Gotisieren unmittelbar vorangehende Zeit und lässt daher auf seine Datierung in die erste Hälfte des 12. Jahrhunderts schlieBen. ${ }^{20}$ Das Krakauer Fragment dürfte somit laut unserer Annahme etwas später entstanden sein, was auch die vergleichende Untersuchung der Notation der beiden Quellen bestätigt.

Der Codex Albensis wurde durchwegs mit linienloser süddeutscher Neumenschrift notiert, ohne Zeichen der Diastematie. Auf dem Fragment sind dagegen zwei Arten von Notation vorhanden, die sowohl aus musikpaläographischer Sicht

16. Knižnica Spišskej kapituly Ms. Mus. Nr. 2, f. 251v-256v. Facsimile-Ausgabe der Handschrift: Spišský antifonár [Das Zipser Antiphonar], hrsg. von Rastislav Adamko - Eva Veselovská - Juraj Šedivý (Ružomberok: Katolícka univerzita), 2008.

17. Istanbul, Topkap Saray, Deissmann 42., f. 299v, 302r. Facsimile-Ausgabe der Handschrift: The Istanbul Antiphonal about 1360, hrsg. von Janka Szendrei (Budapest: Institute for Musicology of the Hungarian Academy of Sciences, 2002) (= Musicalia Danubiana 18).

18. Széchényi Nationalbibliothek Budapest, Fragm. A 58.

19. Universitätsbibliothek Graz 211. Facsimile-Ausgabe der Handschrift: Codex Albensis. Ein Antiphonar aus dem 12. Jahrhundert, hrsg. von Zoltán Falvy - László Mezey (Budapest - Graz: Akadémiai Kiadó - Akademische Druck- und Verlagsanstalt, 1963) (= Monumenta Hungariae Musica 1).

20. „Die in Minuskeln geschriebenen Textpartien [...] erlauben keine andere Datierung als die Mitte oder vielmehr die erste Hälfte des 12. Jahrhunderts.“ Ebd., 18. 
als auch funktional voneinander eindeutig zu trennen sind. Die auf Vierliniensystem gesetzte Hauptschrift mit Schlüsselbuchstaben ohne Custos wurde für die Eintragung von primär musikalischen Gattungen (Antiphonen und Responsorien) verwendet. Bei der Festhaltung von rezitativen Gesängen wie responsoria brevia und Versikel begnügte man sich mit den gewohnten, allerdings diastematisch verlaufenden Neumen in campo aperto (vgl. Abbildung 1). Dass diese Spaltung nicht nachträglich als Ergebnis einer zufallsartigen Entscheidung zustande kam, sondern planmäßig entworfen wurde, bestätigt der Wort- und Zeilenabstand mit entsprechendem Raum für die Neumenzeichen.

Die Hauptschrift des Fragments dürfte unserer Meinung nach das Frühstadium der mittelalterlichen Graner Choralnotation repräsentieren. Die frühesten Vorläufer dieser Notenschrift sind laut Janka Szendrei in den nachträglich, im beginnenden 12. Jahrhundert eingefügten Eintragungen der sog. Hartwick-Agenda bemerkbar. ${ }^{21}$ Ihre erste vollwertige Ausprägung erscheint wiederum in den im ausgehenden 12. Jahrhundert verfassten Notationsweisen des Codex Prayanus. ${ }^{22}$ Im Folgenden wird versucht, den Platz der Notenschrift des Krakauer Fragments innerhalb dieser frühen Entwicklungslinie der Graner Choralnotation zu bestimmen (Tabelle 4).

Die Notenschrift des Krakauer Fragments ist durch zahlreiche archaische Merkmale gekennzeichnet (vgl. Abbildungen 1-2 und Tabelle 4). Die Funktion des syllabischen Grundzeichens ist noch nicht von dem später alleinherrschenden metzer Tractulus, sondern von dem diesem unmittelbar vorangehenden wellenförmigen Punctum erfüllt. Allerdings spielt neben dem Punctum auch die Virga eine ebenso wichtige Rolle, was wohl als Überrest der in den frühesten ungarischen Quellen verwendeten deutschen Neumenschrift anzusehen ist. Die einst konsequent bewahrte Unterscheidung der beiden Grundzeichen - Punctum für den tieferen, Virga für den höheren Ton - scheint jedoch bereits aufgelockert zu sein. Die Virga-Zeichen des Fragments zeigen jene formale Umwandlung, die im Verhältnis zum Punctum von einer entgegengesetzten Richtung die spätere metzer Tractulusform erreicht: Während das Punctum allmählich heranwächst und eine Wellengestalt annimmt, verliert die Virga ihren Schaft, um lediglich ihren Kopf als Notenzeichen zu bewahren. Als Resultat beider Prozesse steht schließlich dieselbe Tractulusform da: das syllabische Grundzeichen der voll entwickelten Graner Choralnotation.

Ähnlich wie die Einzeltöne, zeigt auch die graphische Ausführung der weiteren Neumen einige Besonderheiten, die vom Zeichensystem der reifen Graner Notenschrift abweichen. Vergebens sucht man zum Beispiel nach dem charakteristischen runden Pes der späteren ungarischen Handschriften; statt dessen er-

21. Janka Szendrei, „Die Geschichte der Graner Choralnotation“, Studia Musicologica 30 (1988), 25-28; dies., A „Mos Patriae“ kialakulása 1341 elötti hangjegyes forrásaink tükrében [Die Entwicklung des „Mos Patriae“ in den ungarischen notierten Quellen vor 1341] (Budapest: Balassi Kiadó, 2005), 66-103.

22. Szendrei, „Geschichte der Graner Choralnotation“, 28-32; dies., Die Entwicklung des „Mos patriae“, 144-209. 
Tabelle 4 Der Neumenbestand des Krakauer Fragments

Punctum / Tractulus
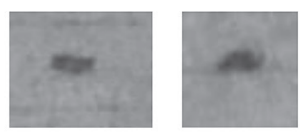

Virga

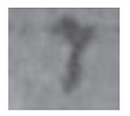

Pes

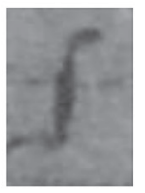

Clivis
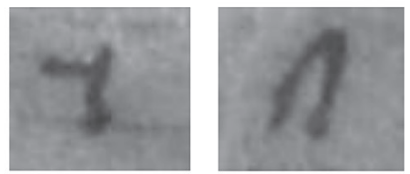

Scandicus

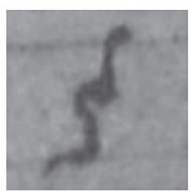

Zusammensetzungen
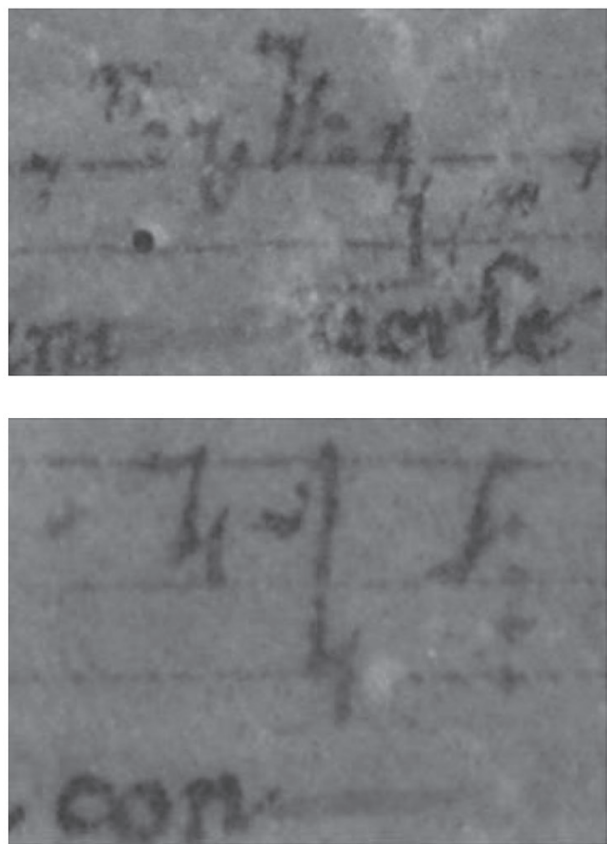
scheint eine etwas asymmetrisch ausgeführte Neume mit einer links angesetzten, wellenförmigen unteren Note und einem nach rechts neigenden oberen Notenkopf. Diese Neumenform passt allerdings strukturell vollkommen ins Zeichensystem der Graner Choralnotation. Neben der vorherrschenden rechtwinkligen Form taucht auf dem Fragment auch die runde deutsche Clivis auf, die in den späteren ungarischen Quellen nicht mehr zu finden ist. Der charakteristische, mit einem Doppelpunkt ansetzende ungarische Climacus kommt zwar nicht vor, vorhanden ist dagegen der gebundene Scandicus sowie die kettenartig aneinandergefügten typischen Neumengruppen mit senkrechten Punktreihen, die zu den auffallenden strukturellen Zeichen der ausgeprägten ungarischen Notation gehören. ${ }^{23}$

Fassen wir die Ergebnisse der musikpaläographischen Untersuchung zusammen, so erscheint die Feststellung nicht übertrieben, dass das Krakauer Fragment als einzigartiger Repräsentant jener Epoche dasteht, in der die formalen Charakteristika des süddeutschen Neumenbestandes noch intensiv bewahrt werden, einzelne Neumenzeichen, strukturelle Eigentümlichkeiten und vor allem Zusammensetzungen aber eine Loslösung von dieser Grundlage und zugleich Zuwendung zu den metzer Formen zeigen. Die Besonderheiten bzw. Anomalien des Zeichensystems sind offensichtlich Archaismen, die größtenteils durch die unmittelbare zeitliche Nähe der linienlosen Neumenschrift zu erklären sind. (Es sei u. a. auf den gemeinsamen Gebrauch von Punctum und Virga hingewiesen - ein Phänomen, das eigentlich nur im linienlosen Raum seine bedeutungsunterscheidende Funktion erfüllen kann.) Aus diesen paläographischen Eigenschaften ist darauf zu schließen, dass die Handschrift kurz nach der Einführung des Liniensystems verfasst wurde: Sie entstand in jener Übergangsperiode, in der die beiden Neumensysteme noch parallel nebeneinander existierten. Obwohl das neue System noch nicht in seiner ausgeprägten Gestalt erscheint, dient es bereits als primäre Notenschrift. Das alte dient wiederum als sekundäre usuelle Schrift bei der Festhaltung jener Gesänge, die später lediglich in den Rubriken aufgelistet werden.

Hypothetisch ist es anzunehmen, dass das Fragment eine Blitzaufnahme der frühesten Phase der Graner Choralnotation repräsentiert. Es dürfte nur wenige Jahre nach dem im ersten Drittel des 12. Jahrhunderts neumierten Codex Albensis entstanden sein, geht aber der ausgeprägten Liniennotation des Codex Prayanus des späten 12. Jahrhunderts einige Jahrzehnte sicher voraus.

\section{Die Demetriusgesänge}

Das Krakauer Fragment stammt aus jener Frühphase der ungarischen Liturgie- und Musikgeschichte, die mit Primärquellen am wenigsten belegt ist. Seine Bedeutung 
liegt jedoch nicht allein im paläographischen Wert. Zugleich ist es die bisher älteste Quelle des Demetriusoffiziums: jenes in Ungarn entstandenen liturgisch-musikalischen Zyklus, dessen textliche sowie musikalische Überlieferung vor 1290 bzw. $1360^{24}$ bisher durch keine unmittelbaren Zeugnisse dokumentiert wurde.

Die Auswahl und Anordnung der auf dem Fragment erhaltenen Gesänge der ersten Vesper und der ersten Nokturn stimmen mit jener Redaktion der Historia überein, die in zwei handschriftlichen ungarischen Brevieren des 15. Jahrhunderts $^{25}$ sowie im gedruckten Breviarium Strigoniense ${ }^{26}$ überliefert ist. Was die erste Vesper anlangt - die hinsichtlich der Variabilität mehr signifikant ist als die beinahe identisch zusammengestellten Nokturnen -, darf ebenso das Antiphonar aus Istanbul hierher gezählt werden: In seinem Anhang sind die Gesänge dieser Hore in der gleichen Anordnung überliefert. Die Texte der Demetrius-Gesänge des Krakauer Fragments fügen sich in die Überlieferungslinie der bisher bekannten Quellen ein, und abgesehen von geringen melodischen Varianten stimmen auch die Melodien mit jenen der entsprechenden Gesänge im Antiphonale Scepusiense und dem Antiphonar aus Istanbul überein. Ein einziger Gesang des Fragments verdient dagegen besondere Aufmerksamkeit (Notenbeispiel 1).

Das Invitatorium Preciosi martyris Demetrii galt als jener Bestandteil der Demetrius-Historia, der neben den allgemeinen musikalisch-stilistischen Merkmalen als einziges ,,beinahe primäres“ Argument die Datierung des Offiziums ins 12. Jahrhundert bestätigen konnte. Wie es bereits in der 2013 veröffentlichten kritischen Edition der Historia ${ }^{27}$ betont wurde, zeigt das Invitatorium eine auffallende melodische Ähnlichkeit mit dem Invitatorium Prestolantes Redemptorem der Adventszeit, das vermutlich dem normannischen Repertoire entnommen und um die Wende des 11.-12. Jahrhunderts in die ungarische Offiziumsliturgie eingeführt wurde. ${ }^{28}$ Die melodische Übereinstimmung ist derart hoch, dass es sich bei den zwei Invitatorien eigentlich um Kontrafakturen handelt. Das Krakauer Fragment liefert nun einen weiteren - und zugleich lustigen - Zeugen der engen Beziehung der beiden Gesänge. Der Schreiber dürfte wohl Text und Melodie von Prestolantes derart in den Ohren gehabt haben, dass er im Schlussteil von Preciosi martyris nach dem in beiden Gesängen vorkommenden Wort quoniam zum

24. Dies sind die Entstehungsjahre der bereits erwähnten frühesten handschriftlichen Belege der Demetrius-Historia. Vgl. Anmerkung 15 und 17 bzw. das Quellenverzeichnis in Tabelle 3.

25. Breviarium aus Bártfa (Bartfeld/Bardejov in der heutigen Ostslowakei), Budapest, Magyar Nemzeti Múzeum (Ungarisches Nationalmuseum) 63.84.C, f. 288r-289v; Breviarium Strigoniense, Alba Iulia, Bibliotheca Batthyanaeum R I 110, f. 362r-363r.

26. Breviarium Strigoniense 1484, f. 353v-354v.

27. Czagány - Tóth, Historia sancti Demetrii, xxxiii.

28. Mehr dazu siehe: Zsuzsa Czagány, „Magyar-normann zenei kapcsolatok a középkorban?“ [Ungarisch-normannische musikalische Beziehungen im Mittelalter?], in Zenetudományi Dolgozatok 1990-1991, hrsg. von László Felföldi - Katalin Lázár (Budapest: Institute for Musicology of the Hungarian Academy of Sciences, 1991), 9-21; dies., „Magyar-normann zenei kapcsolatok a középkorban II.“ [Musikalische Verbindungen zwischen Ungarn und dem Normannenreich im Mittelalter II], in Zenetudományi Dolgozatok 2010, hrsg. von Gábor Kiss (Budapest: Institute for Musicology of the Hungarian Academy of Sciences, 2010), 11-22. 
NotenbeISPIEl 1 Die Invitatorien Prestolantes Redemptorem und Preciosi martyris Demetrii MMMAe $^{29}$
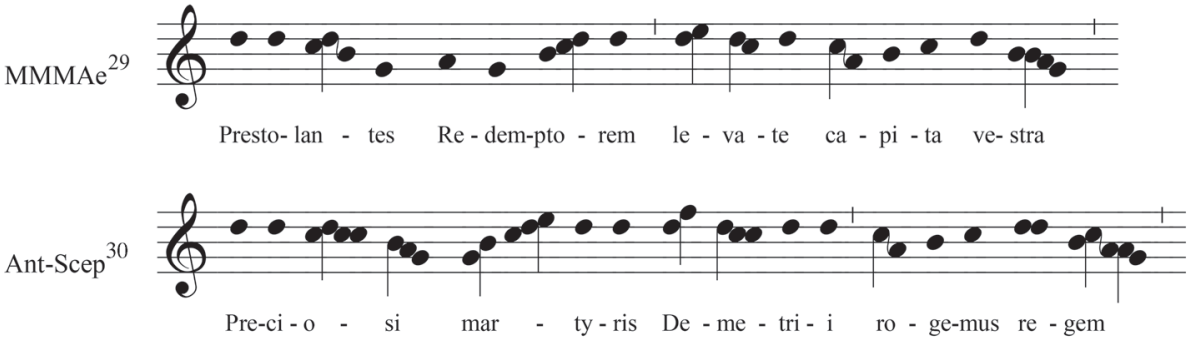

MMMAe

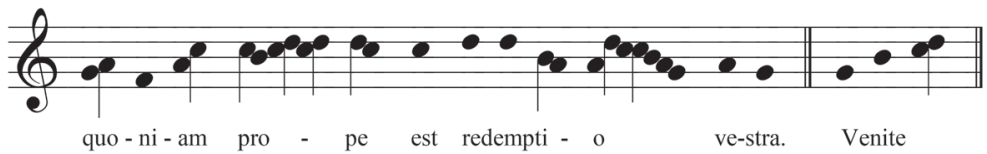

Ant-Scep

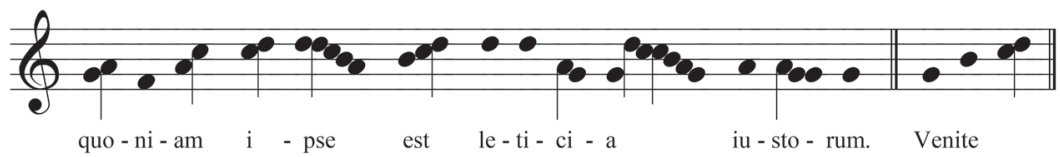

Prestolantes wechselte und das Demetrius-Invitatorium - textlich und melodisch - mit der letzten Zeile von Prestolantes beendete (Notenbeispiel 2).

Sowohl die Notenschrift als auch der Inhalt sprechen demnach dafür, dass das Krakauer Fragment im 12. Jahrhundert, einige Jahre nach dem Codex Albensis entstanden ist. Zur Stärkung dieser Hypothese ist nun ein drittes Argument heranzuführen, mit dem zugleich auf das bereits angesprochene unmittelbare Nacheinanderfolgen der Michaels- und Demetriusoffizien zurückgegriffen wird.

Ähnlich wie die orthodoxen Kalendarien, führen die mittelalterlichen ungarischen Quellen das Demetriusfest konsequent am 26. Oktober. Bei diesem Tag findet sich der Name des Heiligen bereits im Codex Prayanus. ${ }^{31}$ Zwischen dem Michaelsfest am 29. September und dem des Demetrius am 26. Oktober listen die mittelalterlichen ungarischen Kalendarien natülich zahlreiche weitere Feste auf - so etwa jene von Hieronymus, Dionysius, Gereon, Gallus, Ursula mit Gefährtinnen usw. -, darunter auch solche, die bereits in den frühesten Quellen mit Eigengesängen versehen wurden. ${ }^{32}$ Da es sich bei dem Krakauer Fragment um das Fragment eines Antiphonars handelt, kommen nur Feste in Betracht, die, wenn auch nicht unbedingt ein vollständiges Eigenoffizium, so doch wenigstens einige

29. Antiphonen, hrsg. von László Dobszay - Janka Szendrei (Kassel: Bärenreiter, 1999) (= Monumenta Monodica Medii Aevi 5), Nr. 7107.

30. Antiphonale Scepusiense, Knižnica Spišskej kapituly Ms. Mus. Nr. 2., f. 252r.

31. Codex Prayanus, Széchényi Nationalbibliothek Budapest, MNy 1, f. 6r.

32. Vgl. dazu die angeführten Bände der Editionsreihe CAO-ECE. 
Notenbeispiel 2 Die Invitatorien Prestolantes und Preciosi (Zeilen 1-2) und die „kontaminierte“ Fassung des Krakauer Fragments (Zeile 3)

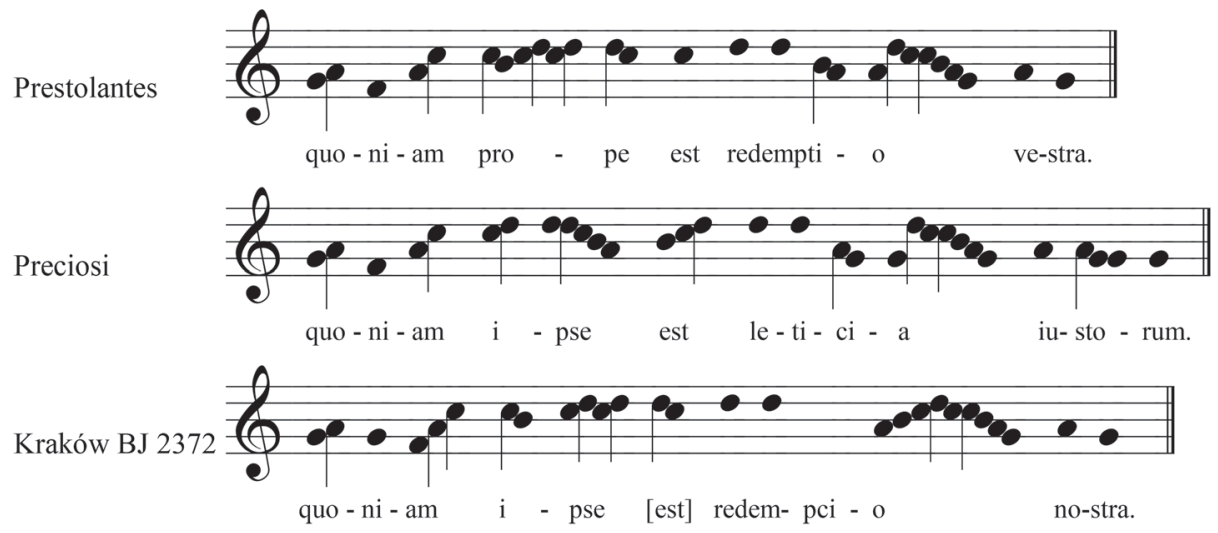

Propriumsgesänge - Benedictus- und Magnificat-Antiphonen, Kommemorationsgesänge - enthalten. Man dürfte etwa nach dem Gallusoffizium fragen, findet sich dieses doch vollständig bereits im zeitlich nahe liegenden Codex Albensis. ${ }^{33}$ Das Offizium wird jedoch auch in den späteren ungarischen Handschriften des zentralen Graner Ritusgebietes eher sporadisch überliefert, ${ }^{34}$ im Gegensatz zu den späteren Brevieren des Ostens (Siebenbürgen und Waradinum) ${ }^{35}$ sowie zu denen aus Kalocsa, ${ }^{36}$ welche eine vollständige Gallushistoria enthalten. Das Fehlen von Gallus kann demnach nicht als ein Hinweis auf die Datierung, wohl aber als ein Hinweis auf die Provenienz des Krakauer Fragments betrachtet werden.

Die Absenz des Offiziums der heiligen Ursula mit ihren elftausend Gefährtinnen kann sich hingegen bei der Datierung des Fragments als hilfreich erweisen. Die späteren ungarischen Handschriften überliefern zwei Offizien zum Fest: Laetis canamus vocibus in den meisten zentralen Quellen aus Esztergom/Gran ${ }^{37}$ bzw. $O$ felix Germania in einigen Brevieren der nördlichen und östlichen Peripherie. ${ }^{38}$ Das Offizium Laetis canamus ist im Codex Albensis noch nicht zu finden, seine ungarische Tradierung beginnt erst mit dem Breviarium Strigoniense MR 67 des 13. Jahrhunderts und bleibt in den späteren Quellen ununterbrochen vorhanden. Das Fehlen des Ursulaoffiziums unterstützt demnach die angenommene, kurz nach der Niederschreibung des Codex Albensis, um die Mitte des 12. Jahrhunderts, gesetzte Entstehungszeit des Fragments.

Fasst man die bisherigen Ergebnisse zusammen, so ist es klar, dass das Krakauer Antiphonarfragment uns unmittelbar in jene Epoche zurückführt, in der die

33. Codex Albensis, f. 123r-125r.

34. CAO-ECE V/B Esztergom, 268.

35. CAO-ECE VII/B Transylvania-Waradinum, 118-119.

36. $C A O-E C E \mathrm{VI} / \mathrm{B}$ Kalocsa-Zagreb, 127-129.

37. $C A O-E C E \mathrm{~V} / \mathrm{B}$ Esztergom, 156-157

38. CAO-ECE V/B Esztergom, 271-272. 
von vielen Seiten beeinflusste junge ungarische Kirche ihr eigenständiges Profil, ihre consuetudo entworfen und festgelegt hat. Es war ebenso die Frühepoche der Konstituierung der ungarischen Choralnotation, in der die Auswirkung der süddeutschen Neumenschrifttypen noch wesentlich stärker zur Geltung kommt. Die wenigen, doch aus musikalischer Sicht umso bedeutsameren Quellen dieser Epoche - u. a. der Codex Albensis, die Hartwick-Agenda, der Codex Prayanus oder das Graner Benedictionale - sind von den neuesten Forschungen erneut aufgegriffen und ins neue Licht gestellt. ${ }^{39}$ Mit seinem erhaltenen Bruchstück des Sanktorale kann vielleicht auch das in Krakau gefundene Fragment zum besseren und differenzierteren Verständnis der Frühgeschichte des mittelalterlichen ungarischen Offiziums und seines Repertoires dienen.

39. Vgl. Szendrei, Die Entwicklung des „Mos Patriae“; Miklós Földváry, Az Esztergomi Benedikcionále. Irodalom és liturgia az államalapítás kori Magyarországon [Das Graner Benedictionale. Literatur und Liturgie in Ungarn in der Zeit der Staatsgründung] (Budapest: ELTE BTK Vallástudományi Központ, Liturgiatörténeti Kutatócsoport, 2014). 